\title{
Sobrevivência de pupas de Lucilia eximia (Wiedemann) (Diptera, Calliphoridae) após submersão em laboratório
}

\author{
Alex Sandro Barros de Souza $^{1} \&$ Ruth Leila Ferreira Keppler ${ }^{1}$
}

${ }^{1}$ Coordenação de Pesquisas em Entomologia, Instituto Nacional de Pesquisas da Amazônia, Campus II, Caixa Postal 478, 69011-970 Manaus-AM, Brasil. alexsouza@inpa.gov.br; ruth@inpa.gov.br

\begin{abstract}
Survival of Lucilia eximia (Wiedemann) (Diptera, Calliphoridae) pupae after submersion in laboratory. Between May and June of 2008, the survival of the pupae of Lucilia eximia (Diptera, Calliphoridae) was observed after submersion in laboratory. For this experiment 480 pupae of same age were used, these were divided in eight groups: the group control was not submerged and the other groups were submerged from one to seven days. The increase of the submersion period reduces the survival, with one day of submersion the survival is of $80 \%$, with two days $40 \%$, of $30 \%$ with three days, in the fourth day it falls to $23,34 \%$ and in the fifth day it is $10 \%$. After this period the mortality rises to $100 \%$. This pattern can be explained by the curve "U-shaped" that happens in the consumption of $\mathrm{O}_{2}$ during the pupal period, where the consumption is larger at the beginning and at the end of the pupal period. The time of submersion also affects the development time, increasing the pupal period. These data are potentially useful in estimating duration of submergence of a corpse in forensic entomology investigations.
\end{abstract}

KEYWORDS. Calliphoridae; consumption of $\mathrm{O}_{2}$; forensic entomology; pupae.

RESUMO. Sobrevivência de pupas de Lucilia eximia (Wiedemann) (Diptera, Calliphoridae) após submersão em laboratório. Entre Maio e Junho de 2008 foi observado a sobrevivência das pupas de Lucilia eximia (Diptera, Calliphoridae) após submersão em laboratório. Para este experimento foram utilizadas 480 pupas de mesma idade, sendo estas divididas em oito grupos: o controle não foi submerso e os demais grupos foram submersos de um a sete dias. $\mathrm{O}$ aumento do período de submersão diminui a sobrevivência, com um dia de submersão a sobrevivência é de $80 \%$, com dois dias $40 \%$, é de $30 \%$ a partir do terceiro dia, no quarto dia cai para $23,34 \%$ e no quinto dia fica em $10 \%$. Após este período a mortalidade sobe para $100 \%$. Este padrão pode ser explicado pela curva "U-shaped" que ocorre no consumo de $\mathrm{O}_{2}$ durante o período pupal, onde o consumo é maior no início e no final do período pupal. O tempo de submersão também afeta o tempo de desenvolvimento, aumentando o período pupal. Estes dados têm o potencial para serem utilizados em investigações envolvendo Entomologia Forense, para a estimativa do tempo de submersão de um cadáver.

PALAVRAS-CHAVE. Calliphoridae; consumo de $\mathrm{O}_{2}$; entomologia forense; pupa.

Entomologia Forense Médico-Legal é a aplicação do estudo de insetos e outros artrópodes associados com cadáver humano em decomposição (Byrd \& Castner 2001). A entomologia forense pode ser utilizada para determinar quando, onde e em que circunstâncias a morte ocorreu, se houve consumo de drogas por parte do falecido, poucas horas antes de sua morte, definir se o cadáver foi retirado e transportado do local original do crime com o objetivo de dificultar uma posterior investigação, ocorrência de traumas em partes específicas do corpo, tempo de decapitação ou retirada de membros do cadáver, além de fornecer informações se a vítima teria sido molestada sexualmente (Campobasso \& Introna 2001; Benecke 2001; Benecke \& Lessig 2001).

Dentre os insetos, os dípteros da família Calliphoridae são os primeiros a localizar o cadáver para realizar a postura dos ovos e a determinação da idade das larvas ou pupas, coletadas no cadáver, indicará o intervalo postmortem (IPM) (OliveiraCosta 2008). Os estágios imaturos são encontradas inclusive em cadáveres parcialmente submersos (Payne \& King 1972; Smith 1986; Tomberlin \& Hadler 1998) e o conhecimento da biologia dos imaturos, neste tipo de ambiente é de extrema importância, pois pode ser estimado o tempo de submersão do cadáver (Merrit \& Wallace 2001).

Estudos com espécies da região Neártica foram realizados para verificar o consumo de oxigênio em pupas de Calliphoridae e sua capacidade de sobrevivência com reduzida demanda de $\mathrm{O}_{2}$ (Hitchock \& Haub 1941; Park \& Buck 1960). Naquela região, apenas um estudo foi conduzido para verificar a sobrevivência de Calliphoridae após submersão na água (Singh \& Greenberg 1994). Lucilia eximia (Wiedemann, 1819) é uma espécie Neotropical (Paraluppi \& Castellon 1994; Mello 2003) e considerada de importância forense no Brasil devido ao grande número de imaturos e adultos que colonizam cadáveres (Salviano et al. 1996; Carvalho \& Linhares 2001; Oliveira-Costa et al. 2001; Souza et al. 2008) é muito importante o conhecimento de sua biologia e fatores que afetem este processo.

Este estudo verifica o efeito da submersão na sobrevivência e no tempo de desenvolvimento de $L$. eximia e o potencial uso destes dados em investigações criminais, visto que pupas de 
Calliphoridae são encontradas aderidas a pele, cabelos e roupas de cadáveres submersos (Singh \& Greenberg 1994; Merrit \& Wallace 2001) e no Brasil não há registro de estudos com este propósito.

Este estudo foi conduzido de maio a junho de 2008 no Laboratório de Biologia de Insetos do Instituto Nacional de Pesquisas da Amazônia (INPA), Manaus, Amazonas.

Para obtenção dos imaturos foram coletadas fêmeas grávidas em carne bovina em decomposição dispostas na área externa da Coordenação de Pesquisas em Entomologia localizado no campus II ( $3^{\circ} 05^{\prime} 41.34$ "S; 59 59' $\left.23.24 " \mathrm{~W}\right)$. Os adultos de $L$. eximia foram identificados com auxílio das chaves de Mello (2003) e Carvalho \& Mello-Patiu (2008).

Após a coleta as fêmeas foram levadas ao laboratório e mantidas à temperatura e umidade relativa de $25 \pm 3^{\circ} \mathrm{Ce} 70 \pm$ $6 \%$, respectivamente, com fotoperíodo de 12 horas. Carne moída bovina em processo de decomposição (retirada da refrigeração 48 horas antes) foi oferecida em placas de Petri como substrato para ovipostura. Quando as larvas de terceiro instar alcançaram o estádio pós-alimentar, ou seja, começaram a abandonar o substrato alimentar, foram transferidas para frascos contendo vermiculita para completarem o desenvolvimento até a fase de pupa, também mantidas as mesmas condições de temperatura, umidade e fotoperíodo das fases anteriores de desenvolvimento.

Para verificar a sobrevivência após submersão, foram utilizadas pupas com três dias de desenvolvimento; estas foram dividas em oitos grupos (cada grupo contendo 30 pupas): GR= grupo controle; G1; G2; G3; G4; G5; G6 e G7. O grupo controle foi mantido em recipientes contendo vermiculita e coberto por voal para completar o desenvolvimento; os demais grupos foram colocados separados em frascos de vidro contendo $30 \mathrm{ml}$ de água destilada. O G1 ficou um dia submerso, o G2 dois dias submerso, assim sucessivamente até completar sete dias de submersão. Quando colocadas nos recipientes contendo água, para evitar a flutuação das pupas, foram colocados pedaços de papel filtro para forçar a submersão das pupas e com o auxílio de pipetas, foram retiradas as bolsas de ar formadas ao redor destas.

Quando as pupas atingiam o tempo estabelecido de submersão, eram retiradas do frasco e acondicionadas em recipientes contendo vermiculita e cobertos com voal para completar o tempo de desenvolvimento. Após este procedimento estes recipientes foram observados diariamente entre $08 \mathrm{~h} 00$ e $21 \mathrm{~h} 00$ com intervalos de uma hora para observação da emergência. Os adultos obtidos foram sacrificados sob temperatura de $-5^{\circ} \mathrm{C}$, uma parte destes foi fixada a seco e outra em álcool a $80 \%$. A parcela fixada a seco foi depositada na Coleção de Invertebrados do INPA. Este experimento foi repetido mais uma vez, obedecendo à mesma metodologia.

O total de indivíduos utilizados e o total de emergidos, juntamente com o tempo de submersão e tempo total de desenvolvimento estão demonstrados na Tabela 1. No grupo controle, $100 \%$ dos adultos emergiram padrão também constatado na réplica. O tempo de submersão está inversamente relacionado com a sobrevivência; quanto maior o tempo de submersão menor é o número de adultos emergidos. As pupas apresentaram capacidade de sobrevivência até o quinto dia de submersão, no sexto e sétimo dia a mortalidade foi de $100 \%$, fato também observado na réplica.

Singh \& Greenberg (1994) trabalhando com cinco espécies de Calliphoridae observaram uma mortalidade de $100 \%$ no quinto dia de submersão para todas as espécies testadas, fato não observado neste experimento, onde a mortalidade alcança este índice no sexto e sétimo dia de submersão. Apesar de não terem trabalhado com L. eximia, Singh \& Greenberg (1994) estudaram Lucilia sericata (Meigen) que é uma espécie do mesmo gênero e possui um tempo de desenvolvimento similar ao de L. eximia (Oliveira-Costa 2008), sendo utilizada como indicadora forense na Europa e Estados Unidos (Greenberg 1991).

O tempo de desenvolvimento também foi afetado pelo tempo de submersão; quanto maior o tempo de submersão maior o tempo de desenvolvimento (Tabela I). Souza et al. (2008) trabalhando com L. eximia no Rio Grande do Sul obtiveram tempo de desenvolvimento de 28,03 dias com temperatura média de $18,34^{\circ} \mathrm{C}$. Greenberg \& Szyska (1984) também trabalhando com L. eximia, obtiveram 22,5 dias para o desenvolvimento total, com $26 \pm 3,1^{\circ} \mathrm{C}$ de temperatura média. Fraga (2004) estudando o comportamento de ovipostura e tempo de desenvolvimento de L. eximia em uma reserva florestal em Manaus, verificou que na estação seca o tempo de desenvolvimento total foi de 17,6 dias e na estação chuvosa foi de 19,9 dias. Neste estudo, o grupo controle, apresentou um tempo médio de desenvolvimento de 20 dias, fato também observado na réplica. Entretanto este tempo aumenta conforme aumenta o período de submersão (Tabela I), como pode ser observado em pupas com cinco dias de submersão em que o tempo total de desenvolvimento em média foi de 25 dias para o experimento e de $25,5 \pm 0,7$ dias para a réplica.

Kalmus (1937) observou que em ambientes com baixa demanda de O2 o período pupal em Drosophila (Diptera, Drosophilidae) tende a se prolongar. Estes dados podem explicar o aumento no tempo de desenvolvimento neste estudo; outro fator que pode explicar esta variação é o consumo variado de oxigênio que ocorre no período pupal, como constatado por Dobzhansky \& Poulson (1935) quando utilizaram Drosophila pseudoobscura, demonstrando que o consumo de oxigênio é alto no início do processo pupal e depois cai drasticamente até chegar ao valor mínimo, permanecendo constante durante alguns dias e subindo novamente quando está perto da emergência. Nesta fase, o consumo de oxigênio atinge valores iguais aos do início do processo pupal. Este padrão, em que o consumo de $\mathrm{O} 2$ é maior no início e no final do período pupal é denominado curva "Ushaped", o que confere uma habilidade de sobreviver em hipoxia, por parte destes dípteros. Em Calliphoridae este comportamento foi demostrado em Phormia regina (Meigen) por Hitchock \& Haub (1941), Keister (1953) e Park \& Buck (1960) e em Lucilia sericata por El-Kady et al. (1999).

A potencial aplicabilidade destes dados está envolvida em investigações criminais onde há necessidade da estimativa do tempo de submersão de um cadáver. Assim em cadáveres onde ocorreu a fixação de pupas antes de sua total submersão; o tempo de submersão pode ser estimado de duas formas: 1 . Períodos em que a mortalidade dos adultos é $100 \%$, pode-se estimar que este corpo passou por um período superior a cinco dias de submersão, fato que impediu a emergência de adultos, 
Tabela I. Tempo de submersão; número de adultos emergidos e tempo de desenvolvimento de Lucilia eximia em laboratório.

\begin{tabular}{ccccc}
\hline $\begin{array}{c}\text { Tempo de } \\
\text { submersão } \\
\text { (Dias) }\end{array}$ & N & Adultos. & $\begin{array}{c}\text { Tempo de } \\
\text { Desenvolvimento } \\
\pm \text { DP (dias) }\end{array}$ & $\begin{array}{c}\text { Sobrevivência } \\
(\%)\end{array}$ \\
\hline Controle & & & & \\
(não submerso) & 30 & 30 & $20 \pm 0,70$ & $100 \%$ \\
1 & 30 & 24 & $21 \pm 1$ & $80 \%$ \\
2 & 30 & 12 & $22 \pm 1$ & $40 \%$ \\
3 & 30 & 9 & $23 \pm 1$ & $30 \%$ \\
4 & 30 & 7 & $24,5 \pm 0,70$ & $23,34 \%$ \\
5 & 30 & 3 & $25 \pm 1$ & $10 \%$ \\
6 & 30 & 0 & - & $0 \%$ \\
7 & 30 & 0 & - & $0 \%$ \\
Controle R & & & & $100 \%$ \\
(não submerso) & 30 & 30 & $20 \pm 0,70$ & $70 \%$ \\
$1 \mathrm{R}$ & 30 & 21 & $21 \pm 1$ & $46,67 \%$ \\
2 R & 30 & 14 & $22,5 \pm 0,70$ & $23,34 \%$ \\
$3 \mathrm{R}$ & 30 & 7 & $23,5 \pm 0,70$ & $26,67 \%$ \\
$4 \mathrm{R}$ & 30 & 8 & $24 \pm 1$ & $0 \%$ \\
$5 \mathrm{R}$ & 30 & 4 & $25,5 \pm 1$ & $13,34 \%$ \\
$6 \mathrm{R}$ & 30 & 0 & - & $0 \%$ \\
$7 \mathrm{R}$ & 30 & 0 & - &
\end{tabular}

DP= Desvio padrão; N= Número de pupas; $\mathrm{R}=$ Réplica.

2. Períodos em que seja possível a emergência de adultos, partindo da premissa de que quanto maior o período de submersão maior é a duração do período pupal, pode ser estimado o tempo mínimo que este cadáver esteve submerso, estas informações combinadas com dados referntes aos outros estádios de desenvolvimento, auxiliarão para que o cálculo do IPM seja cada vez mais preciso.

Estes dados demonstram a necessidade de um maior conhecimento dos fatores que interferem no tempo de desenvolvimento das espécies envolvidas na decomposição de cadáveres no Brasil. E as amostras utilizadas de L. eximia por estarem na região amazônica, uma região com altos índices pluviométricos (Fisch et al. 1998), podem ter desenvolvido uma habilidade superior às demais espécies de Calliphoridae estudadas, podendo suportar um período maior de submersão. Mas esta hipótese só poderá ser confirmada com estudos posteriores, pois esta relação não foi investigada neste trabalho.

Agradecimentos. Ao CNPq pela concessão da bolsa de mestrado ao autor sênior; a Beatriz Ronchi Telles (Coordenadora do Programa de Pós-graduação em Entomologia-INPA) pelo apoio logístico.

\section{REFERÊNCIAS}

Benecke, M. 2001. A brief history of forensic entomology. Forensic Science International 120: $2-14$

Benecke, M. \& R. Lessig. 2001. Child neglect and forensic entomology. Forensic Science International 120: 155-159.

Byrd, J. H. \& J. L. Castner. 2001 (eds.). Forensic Entomology: The utility of arthropods in legal investigations. Boca Raton, Florida, CRC Press LLC, 418 p.

Campobasso, C. P. \& F. Introna. 2001. The forensic entomologist in the context of the forensic pathologist's role. Forensic Science International 120: 132-139.

Carvalho, C. J. B. de \& C. A. Mello-Patiu. 2008. Key to the adults of the most common forensic species of Diptera in South America.
Revista Brasileira de Entomologia 52: 390-406.

Carvalho, L. M. L. \& A. X. Linhares. 2001. Seasonality of insect succession and pig carcass decomposition in a natural forest area in southeastern Brazil. Journal Forensic Science 46: 604-608.

Dobzhansky T. \& D. F. Poulson. 1935. Oxygen consumption of Drosophila pupae. Journal of Comparative Physiology A: Neuroethology, Sensory, Neural, and Behavioral Physiology 22: $473-478$

El-Kady, E. M.; A. M. Kheirallah; A. N. Kayed; S. I. Dekinesh \& Z. A. Ahmed. 1999. The bioenergetics of the house fly Musca domestica vicina and blowfly Lucilia sericata. Pakistan Journal of Biological Sciences 2: 472-477.

Fisch, G.; J. A. Marengo \& C. A. Nobre. 1998. Uma revisão geral sobre o clima da Amazônia. Acta Amazônica 28: 101-126.

Fraga, N. J. 2004. Comportamento de oviposição de adultos, tempo de desenvolvimento e morfologia dos imaturos de Hemilucilia segmentaria (Fabricius), H. semidiaphana (Rondani) e Lucilia eximia (Wiedemann) (Diptera: Calliphoridae) na Reserva Florestal Adolpho Ducke, Manaus, Brasil. Dissertação, Mestrado, Instituto Nacional de Pesquisas da Amazônia, Manaus. xiii+71 p

Greenberg, B. \& L. M. Szyska. 1984. Immature stages and biology of fifteen species of Peruvian Calliphoridae. Annals of the Entomological Society of América 77: 488-515.

Greenberg, B. 1991. Flies as forensic indicators. Jounal of Medical Entomology 28: 565-577.

Hitchock, F. A. \& J. G. Haub. 1941. The interconversion of food stffus in the blow fly (Phormia regina) during metamorphosis. I. Respiratory metabolism and nitrogen excretion. Annals of the Entomological Society of America 34: 17-25.

Kalmus, H. 1937. Die Entwicklungsdauer von drosophilapuppen bei verschiedener Sauerstofftension. Journal of Comparative Physiology A: Neuroethology, Sensory, Neural, and Behavioral Physiology 24: 409-412.

Keister, M. L. 1953. Some observations on pupal respiration on Phormia regina. Journal of Morphology 93: 573-587.

Mello, R. P. 2003. Chave para identificação das formas adultas das espécies da família Calliphoridae (Diptera, Brachycera, Cyclorrhapha) encontradas no Brasil. Entomologia y Vectores 10: $255-268$.

Merrit, R. W. \& J. R. Wallace. 2001. The role of aquatic insects in forensic ivestigations. p. 177-222. In: Byrd, J. H. \& J. L. Castner (eds.). Forensic Entomology: The utility of arthropods in legal investigations. CRC Press LLC, Boca Raton, Florida.

Oliveira-Costa, J.; C. A. Mello-Patiu \& S. M. Lopes. 2001. Dípteros muscóideos associados com cadáveres humanos no local da morte, no estado do Rio de Janeiro, Brasil. Boletim do Museu Nacional, Série Zoologia 464: 1-6.

Oliveira-Costa, J. 2008. Entomologia Forense: Quando os insetos são vestígios. Campinas Editora Millenium, $420 \mathrm{p}$.

Paraluppi, N. D. \& E. G. Castellon. 1994. Calliphoridae (Diptera) em Manaus: I. Levantamento Taxônomico e Sazonalidade. Revista Brasileira de Entomologia 34: 661-668.

Park, H. D. \& J. Buck. 1960. The relation of oxygen consuption to ambient oxygen concentration during metamorphosis of the blow fly (Phormia regina). Journal of Insect Physiology 4: 220-228.

Payne, J. A. \& E. W. King. 1972. Insect succession and decomposition of pig carcasses in water. Journal of the Georgia Entomological Society 7: $153-162$.

Salviano, R. J. B.; R. P. Mello; R. F. S Beck \& A. Ferreira. 1996. Calliphoridae (Diptera) associated with human corpses in Rio de Janeiro, Brazil. Entomologia y Vectores 3: 145-146.

Singh, D. \& B. Greenberg. 1994. Survival after submergence in the pupae of five species of blow flies (Diptera: Calliphoridae). Journal of Medical Entomology 31: 757-759.

Smith, K. G. V. 1986. A Manual of Forensic Entomology. Cornell University Press, Ithaca, New York, 205 p.

Souza, A. S. B.; F. D. Kirst \& R. F. Krüger. 2008. Insects of forensic importance from Rio Grande do Sul State in Southern Brazil. Revista Brasileira de Entomologia 52: 641-646.

Tomberlin, J. K. \& P. Hadler. 1998. Seasonal colonization and decomposition of rat carrion in water and land in a open field in South Carolina. Journal of Medical Entomology 35: 704-709.

Recebido em 14/08/2008; aceito em 20/01/2009 